\title{
Analysis of treatment outcomes for primary tonsillar lymphoma
}

Yun Hee Lee, MD', Seok Goo Cho, MD, PhD², Seung Eun Jung, MD, PhD ${ }^{3}$, Sung Hoon Kim, MD, PhD ${ }^{4}$ Joo Hyun O, MD, PhD ${ }^{4}$, Gyeong Sin Park, MD, PhD ${ }^{5}$, Suk Woo Yang, MD, PhD ${ }^{6}$, In Seok Lee, MD, PhD ${ }^{7}$, Chin Kook Rhee, MD, PhD7, Byung Ock Choi, MD, PhD ${ }^{8}$

'Department of Radiation Oncology, Gyeongsang National University Hospital, Gyeongsang National University School of Medicine, Jinju; Departments of ${ }^{2}$ Hematology, ${ }^{3}$ Radiology, ${ }^{4}$ Nuclear Medicine, ${ }^{5}$ Pathology,${ }^{6}$ Ophthalmology, ${ }^{7}$ Internal Medicine, and ${ }^{8}$ Radiation Oncology, Catholic University Lymphoma Group (CULG), Seoul St. Mary's Hospital, College of Medicine, The Catholic University of Korea, Seoul, Korea

Purpose: Although each Waldeyer's ring sub-site is considered an independent prognostic factor, few studies have assessed the prognosis and treatment of tonsillar lymphoma. Treatment outcomes were analyzed in patients with primary tonsillar lymphoma who were treated with chemotherapy and radiotherapy (RT).

Materials and Methods: Nineteen patients with diffuse large B-cell lymphoma were evaluated, with a median follow-up of 53 months. Age, sex, and histology, amongst other factors, were reviewed. Progression-free survival (PFS) and overall survival (OS) rates were analyzed.

Results: Most patients had Ann Arbor stage I-II (94.7\%), IPI score of 0 (89.5\%), and complete remission after chemotherapy (89.5\%). The 5-year PFS and OS rates were 74.6\% and 80\%, respectively. In univariate analysis, the rituximab, cyclophosphamide, doxorubicin, vincristine, and prednisone (R-CHOP) regimen resulted in a better PFS than the cyclophosphamide, doxorubicin, vincristine, and prednisone (CHOP) regimen ( $88.9 \%$ vs. 50.0\%; $p=0.053$ ). RT dose was related to the survival outcome ( $p=0.010$ for PFS, $p=0.044$ for OS). Patients were classified into the CHOP + RT ( $>40$ Gy) group and R-CHOP + RT ( $\leq 40$ Gy) group. The 5 -year PFS rates were 50\% in the CHOP + RT group, and $100 \%$ in the R-CHOP + RT group ( $p=0.018)$. The 5 -year OS rates were $66.7 \%$ and $100 \%$, respectively $(p=0.087)$.

Conclusion: Primary tonsillar lymphoma patients typically have favorable outcomes. Chemotherapy (R-CHOP) combined with relatively lower dose consolidative RT may be safe and effective for primary tonsillar lymphoma.

Keywords: Palatine Tonsil, Non-Hodgkin Iymphoma, Radiotherapy

\section{Introduction}

The incidence of non-Hodgkin lymphoma ( $\mathrm{NHL}$ ) involving the Waldeyer's ring (WR) has been increasing in Asia [1]. The
WR includes the pharyngeal (adenoids), tubal, palatine and lingual tonsils (base of the tongue), and WR lymphoma most frequently occurs in the palatine tonsil [1,2]. The location of disease is considered an important prognostic factor for

Received 3 May 2016, Revised 12 July 2016, Accepted 22 August 2016.

Correspondence: Byung Ock Choi, MD, PhD, Department of Radiation Oncology, Seoul St. Mary's Hospital, College of Medicine, The Catholic University of Korea, 222 Banpo-daero, Seocho-gu, Seoul 06591, Korea. Tel: +82-2-2258-1526, Fax: +82-2592-1532, E-mail: choibo67@catholic.ac.kr

(C) This is an Open Access article distributed under the terms of the Creative Commons Attribution Non-Commercial License (http://creativecommons.org/ licenses/by-nc/4.0/) which permits unrestricted non-commercial use, distribution, and reproduction in any medium, provided the original work is properly cited.

www.e-roj.org 
primary extranodal lymphomas [3]. Each of the WR subsites are considered to be independent prognostic factors, and the International Lymphoma Radiation Oncology Group recommends that it is not necessary to treat the entire WR in case of primary tonsillar lymphoma [4]. The majority of reports on tonsillar lymphoma include all WR lymphoma cases; thus, it is difficult to define the treatment results, prognostic factors, and the optimum radiotherapy (RT) courses for primary tonsillar lymphoma $[5,6]$. Furthermore, there is controversy regarding the prognosis of primary tonsillar lymphoma, which requires further evaluation.

Chemotherapy combined with RT is considered the standard treatment for limited-stage diffuse large B-cell lymphoma (DLBCL) since a report by the Southwest Oncology Group (SWOG) 8736 trial [7]. Several studies have shown that RT prolongs progression-free survival (PFS) [8,9]. However, in longterm follow-up studies involving the use of rituximab plus cyclophosphamide, doxorubicin, vincristine, and prednisone (R-CHOP), the role of RT requires clarification [10-12]. With regard to tonsillar lymphoma, a few studies have reported that higher radiation doses result in better local control and overall survival (OS). However, the therapeutic effects of RT and the optimal RT dose require further evaluation with respect to tonsillar lymphoma $[2,13,14]$.

We reviewed patients treated with chemotherapy combined with consolidative RT, and the prognosis and treatment outcomes were analyzed.

\section{Materials and Methods}

We retrospectively reviewed patients treated with chemotherapy combined with RT for primary tonsillar lymphoma between September 1993 and December 2014 at Seoul St. Mary's Hospital. A total of 19 patients were included in the study. Inclusion criteria were as follows: 1) the predominant location of the tumor was the palatine tonsil; 2) the tumor was pathologically confirmed as Iymphoma; 3) histology was classified according to the World Health Organization classification, and diffuse large B cell lymphoma (DLBCL) was included; 4) the tumor was Ann Arbor stage I-III; 5) the tumor was treated with chemotherapy combined with $\mathrm{RT} ; 6)$ the response to chemotherapy was complete or partial remission; 7) RT was prescribed for consolidative purposes; and 8) the patient was followed-up for at least 3 months after RT. Patients were classified according to the Ann Arbor staging system. To evaluate each patient's characteristics, including age, sex, Eastern Cooperative Oncology Group (ECOG) performance status, comorbidity, presenting symptoms, B symptoms (fever, night sweats, and weight loss), initial lactate dehydrogenase (LDH) levels, international prognostic index (IPI) score, Ann Arbor stage, and histologic findings were reviewed. A tumor more than $5 \mathrm{~cm}$ in diameter was defined as bulky disease. Chemotherapy regimen, number of chemotherapy cycles, surgery type, RT dose, RT field, and RT technique were also examined.

PFS and OS were analyzed. PFS was calculated as the period from the first day of chemotherapy to the date of disease progression or death due to any cause. OS was calculated as the period from the first day of chemotherapy to the date of death due to any cause. PFS and OS were analyzed using the Kaplan-Meier method, and prognostic factors were compared using the log-rank test in univariate analysis. A p-value of $<0.05$ was considered statistically significant. All statistical analyses were performed using SPSS ver. 20 (IBM Co., Armonk, NY, USA). The acute and late toxicities related to RT were graded according to the Common Terminology Criteria for Adverse Events (CTCAE) ver. 4.0. This study was approved by the Institutional Review Board of Seoul St. Mary's Hospital.

\section{Results}

\section{Patients and treatment characteristics}

A total of 19 patients with primary tonsillar lymphoma were evaluated in this study. The patient characteristics and treatments administered are shown in Table 1. The age of the patients ranged between 26 and 79 years, with a median of 51 years. The most common presenting symptoms were sore throat (57.9\%) and neck mass (42.1\%). Tonsillectomy for pathologic diagnosis was performed for 5 patients (26.3\%). All patients received combined treatment with chemotherapy and RT. Chemotherapy was administered prior to RT. The chemotherapy regimens included cyclophosphamide, doxorubicin, vincristine, and prednisone (CHOP) in 6 patients (31.6\%) and R-CHOP in 13 patients (68.4\%). The median number of chemotherapy cycles was 6 (range, 1 to 8 ). The majority of patients (89.5\%) showed complete remission after chemotherapy. RT was delivered using a megavoltage linear accelerator. The two-dimensional (2D) RT technique was used in 4 patients who had been treated before 1999 Eleven patients were treated with the three-dimensional (3D) RT technique, and 4 patients were treated with intensitymodulated radiotherapy (IMRT). Both the WR region and neck lymph nodes (LNs) were included in the RT field. RT was administered to the WR region or tonsillar area with a median 
Table 1. Patient and treatment characteristics

\begin{tabular}{|c|c|}
\hline Characteristic & Value \\
\hline \multicolumn{2}{|l|}{ Sex } \\
\hline Male & $12(63.2)$ \\
\hline Female & 7 (36.8) \\
\hline Age (yr) & $51(26-79)$ \\
\hline \multicolumn{2}{|l|}{ ECOG performance status } \\
\hline 0 & $9(47.4)$ \\
\hline 1 & $10(52.6)$ \\
\hline \multicolumn{2}{|l|}{ Comorbidity } \\
\hline Yes & $6(31.6)$ \\
\hline No & $13(68.4)$ \\
\hline \multicolumn{2}{|l|}{ Ann Arbor stage } \\
\hline । & $3(15.8)$ \\
\hline$\|$ & 15 (78.9) \\
\hline III & $1(5.3)$ \\
\hline \multicolumn{2}{|l|}{ B symptom } \\
\hline No & $19(100)$ \\
\hline Yes & $0(0)$ \\
\hline \multicolumn{2}{|l|}{ Initial LDH } \\
\hline Normal & $13(68.4)$ \\
\hline Elevated & $5(26.3)$ \\
\hline Unknown & $1(5.3)$ \\
\hline \multicolumn{2}{|l|}{$|P|$} \\
\hline 0 & $17(89.5)$ \\
\hline 1 & $1(5.3)$ \\
\hline 2 & $1(5.3)$ \\
\hline \multicolumn{2}{|l|}{ Chemotherapy regimen } \\
\hline $\mathrm{CHOP}$ & $6(31.6)$ \\
\hline $\mathrm{R}-\mathrm{CHOP}$ & $13(68.4)$ \\
\hline \multicolumn{2}{|l|}{ Chemotherapy response } \\
\hline Complete remission & $17(89.5)$ \\
\hline Partial remission & $2(10.5)$ \\
\hline \multicolumn{2}{|l|}{ RT technique } \\
\hline $2 \mathrm{D}$ & $4(21.1)$ \\
\hline $3 D$ & $11(57.9)$ \\
\hline IMRT & $4(21.1)$ \\
\hline RT dose, tonsil (Gy) & $36(30-55.8)$ \\
\hline$>40$ & 7 (36.8) \\
\hline$\leq 40$ & $12(63.2)$ \\
\hline RT dose, neck (Gy) & $30.6(26-50.4)$ \\
\hline$>40$ & 7 (36.8) \\
\hline$\leq 40$ & $12(63.2)$ \\
\hline
\end{tabular}

Values are presented as number of patients (\%) or median (range). ECOG, Eastern Cooperative Oncology Group; LDH, lactate dehydrogenase; IPI, international prognostic index; $\mathrm{CHOP}$, cyclophosphamide, doxorubicin, vincristine and prednisone; R-CHOP, rituximab, cyclophosphamide, doxorubicin, vincristine, and prednisone; $R T$, radiotherapy; 2D, two-dimensional; 3D, three-dimensional; IMRT, intensity-modulated radiotherapy.

dose of 36 Gy (range, 30 to $55.8 \mathrm{~Gy}$ ) per 1.8-2 Gy fraction size, and to neck lymph nodes with a median of 30.6 Gy (range,

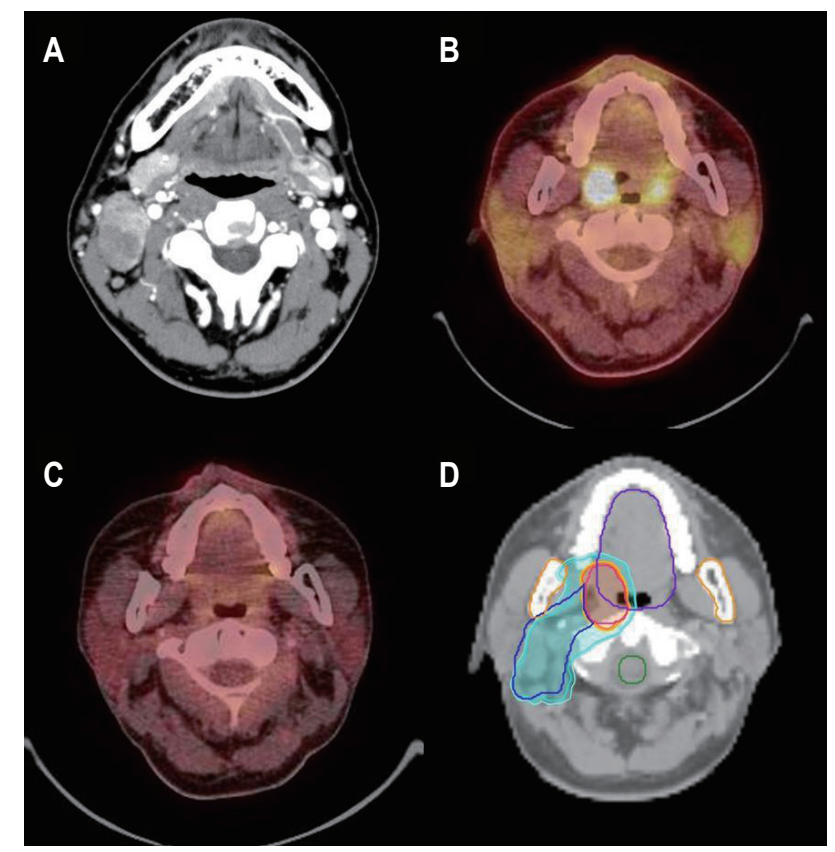

Fig. 1. Intensity-modulated radiotherapy for the primary tonsillar lymphoma. A 37-year-old man presented with a right neck mass. (A) Initial neck computed tomography scan shows multiple lymph node enlargements in the right neck level II. Diffuse large B-cell lymphoma was confirmed by excisional biopsy. (B) High fludeoxyglucose uptake is noted in the right palatine tonsi (SUVmax, 22.8), and postoperative changes are seen in the right neck on positron emission tomography, which confirm a diagnosis of Ann Arbor stage II tonsillar lymphoma. (C) He was treated with 6 cycles of R-CHOP chemotherapy and had complete remission. (D) Consolidative radiotherapy was delivered to the primary tonsillar lesion (red line) with $36 \mathrm{~Gy} / 20$ fractions for 4 weeks (orange color) and to the right neck lymph nodes (blue line) with 30 Gy/20 fractions for 4 weeks (light blue color). SUVmax, maximum standardized uptake value; R-CHOP, rituximab, cyclophosphamide, doxorubicin, vincristine, and prednisone.

26 to 50.4 Gy) per 1.5-2 Gy fraction size. Fig. 1 displays the IMRT planning for 1 patient who had complete remission after receiving $\mathrm{R}$-CHOP chemotherapy for the treatment of Ann Arbor stage II tonsillar lymphoma.

\section{Treatment outcome}

After a median follow-up of 53 months (range, 7 to 249 months), there was no evidence of disease in any of the 15 survivors. Three incidences of recurrence were observed during the follow-up; 2 patients had regional (neck LN) failure, and the remaining patient had distant failure in the abdominalpelvic $L N$ and vagina. The regional failure sites had previously been included in the RT field. The 5-year locoregional control rate was $88.1 \%$. Univariate analysis was not performed 
Table 2. Univariate analysis or prognostic factor of survival outcome

\begin{tabular}{|c|c|c|c|c|c|}
\hline Characteristic & No. of patients & 5-yr PFS (\%) & $p$-value & $5-y r$ OS (\%) & $p$-value \\
\hline Sex & & & 0.735 & & 0.620 \\
\hline Male & 12 & 76.2 & & 75 & \\
\hline Female & 7 & 71.4 & & 85.7 & \\
\hline Age (yr) & & & 0.885 & & 0.399 \\
\hline$<50$ & 8 & 72.9 & & 71.4 & \\
\hline$\geq 50$ & 11 & 77.1 & & 87.5 & \\
\hline ECOG performance status & & & 0.054 & & 0.132 \\
\hline 0 & 9 & 100 & & 100 & \\
\hline 1 & 10 & 55.6 & & 66.7 & \\
\hline Comorbidity & & & 0.264 & & 0.604 \\
\hline Yes & 6 & 53.3 & & 75 & \\
\hline No & 13 & 81.8 & & 81.8 & \\
\hline Initial LDH & & & 0.315 & & 0.600 \\
\hline Normal & 13 & 77.8 & & 77.8 & \\
\hline Elevated & 5 & 60 & & 80.0 & \\
\hline Chemotherapy regimen & & & 0.053 & & 0.258 \\
\hline $\mathrm{CHOP}$ & 6 & 50.0 & & 66.7 & \\
\hline $\mathrm{R}-\mathrm{CHOP}$ & 13 & 88.9 & & 88.9 & \\
\hline RT technique & & & 0.143 & & 0.538 \\
\hline $2 \mathrm{D}$ & 4 & 50.0 & & 75.0 & \\
\hline $3 \mathrm{D}+\mathrm{IMRT}$ & 15 & 82.5 & & 81.8 & \\
\hline RT dose (Gy) & & & 0.010 & & 0.044 \\
\hline$>40$ & 7 & 42.9 & & 57.1 & \\
\hline$\leq 40$ & 12 & 100 & & 100 & \\
\hline
\end{tabular}

PFS, progression free survival; OS, overall survival; ECOG, Eastern Cooperative Oncology Group; LDH, lactate dehydrogenase; CHOP, cyclophosphamide, doxorubicin, vincristine, and prednisone; R-CHOP, rituximab, cyclophosphamide, doxorubicin, vincristine, and prednisone; RT, radiotherapy; 2D, two-dimensional; 3D, three-dimensional; IMRT, intensity-modulated radiotherapy.

because of the small number of cases of locoregional recurrences.

The 5-year PFS and OS rates were $74.6 \%$ and $80 \%$ (Fig. 2A and B). Univariate analysis was performed for PFS and OS (Table 2). The R-CHOP regimen generally resulted in a better PFS than the $\mathrm{CHOP}$ regimen ( $88.9 \%$ vs. $50.0 \% ; p=0.053$ ). RT dose was related to the survival outcome ( $p=0.010$ for PFS and $p=$ 0.044 for 0S). Other factors, including sex, age, comorbidities, initial LDH level, ECOG performance status, and RT technique, had no influence on PFS and OS. The majority of patients had Ann Arbor stage II disease (78.9\%) and an IPI score of 0 (89.5\%), which were not included in the univariate analysis. Multivariate analysis was not conducted because of the small number of patients included. One patient received one cycle of R-CHOP chemotherapy due to poor general condition, and RT was prescribed at a dose of $>40 \mathrm{~Gy}$. Once the aforementioned patient had been excluded, the patients who were treated with the CHOP regimen received a higher RT dose (median, $50.4 \mathrm{~Gy}$ ) than those treated with the R-CHOP regimen (median, 33.3 Gy). The patients treated with the CHOP regimen followed by
RT were treated between 1993 and 2001, and patients in the R-CHOP + RT group were treated between 2009 and 2015. Subsequent to the adjustment in treatment strategy, the patients were classified into two groups: $\mathrm{CHOP}+\mathrm{RT}$ ( $>40 \mathrm{~Gy}$ ) group and R-CHOP + RT ( $\leq 40 \mathrm{~Gy}$ ) group. The 5-year PFS rates were $50 \%$ in the CHOP + RT group and 100\% in the R-CHOP $+\mathrm{RT}$ group $(p=0.018)$, as shown Fig. $2 \mathrm{C}$, and the 5 -year OS rates were $66.7 \%$ and $100 \%$, respectively ( $p=0.087)$, as shown Fig. 2D.

Most patients showed a mild degree of acute oropharyngeal mucositis and xerostomia during treatment with RT. Of the CHOP + RT patients, 5 patients (83.3\%) experienced grade 2 oropharyngeal mucositis and 2 patients (33.3\%) had grade 2 xerostomia. Grade 2 mucositis and xerostomia developed in 5 patients (38.5\%) and 3 patients $(23.1 \%)$, respectively, in the $\mathrm{R}-\mathrm{CHOP}+\mathrm{RT}$ group. There was no difference of acute toxicity rate between the two groups. Grade 2 xerostomia persisted in 1 patient (16.7\%) and 2 patients (15.4\%) in the CHOP + $\mathrm{RT}$ and the R-CHOP + RT groups, respectively. There were no grade 3 or higher toxicities associated with RT. Hypothyroidism 
was observed in 1 patient in the CHOP + RT group at 29 months after RT. One patient developed a second malignancy (Kaposi's sarcoma) in the left base of the tongue (previous radiation field) at 39 months after $\mathrm{RT}$. The patient was treated with 1 cycle of R-CHOP chemotherapy and then RT (45 Gy/25 fraction) on the left tonsillar lesion and left neck node for tonsillar lymphoma. The patient died 1.5 months after the diagnosis of the second malignancy.

\section{Discussion and Conclusion}

In this study, we evaluated the treatment outcomes of patients with primary tonsillar lymphoma who were treated with chemotherapy in combination with RT. The majority of patients presented with early-stage disease. After a relatively long-term follow up, our patients showed a 5-year PFS rate of 74.6\% and a 5 -year OS rate of $80 \%$, which is comparable to the results of previous reports (61\% and 67\%, respectively) [15]. R-CHOP combined with low-dose consolidative RT resulted in a better treatment outcome than CHOP combined with high-dose consolidative RT.

Chemotherapy combined with RT has been considered the standard treatment for limited-stage NHL since the SWOG 8736 study. Although there is controversy regarding the consolidative role of RT, several studies have shown that the addition of RT to chemotherapy improves PFS for patients
A

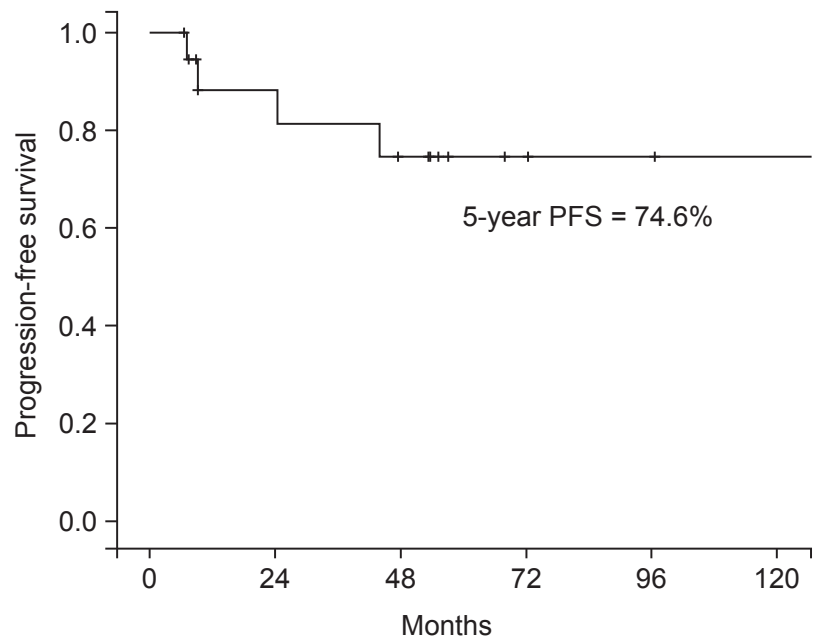

C

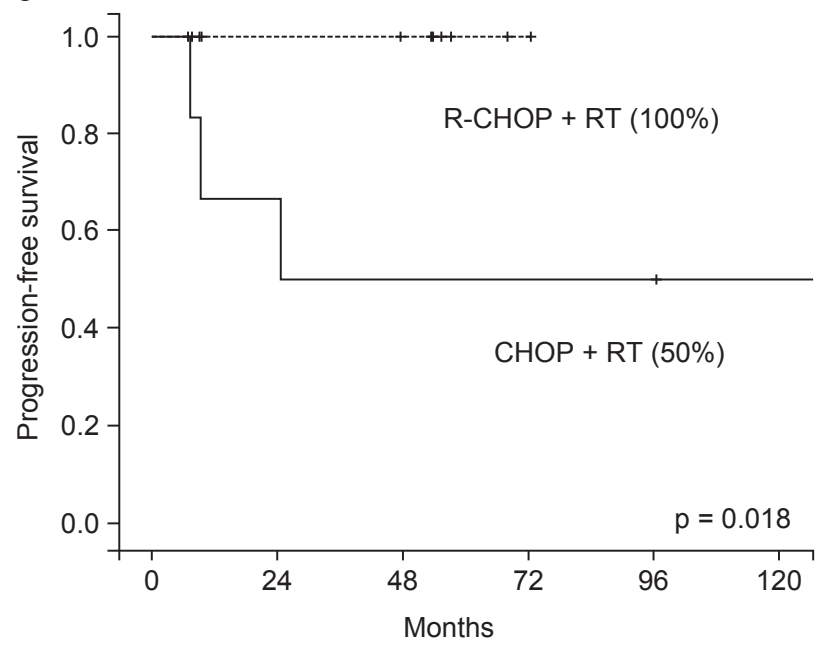

B

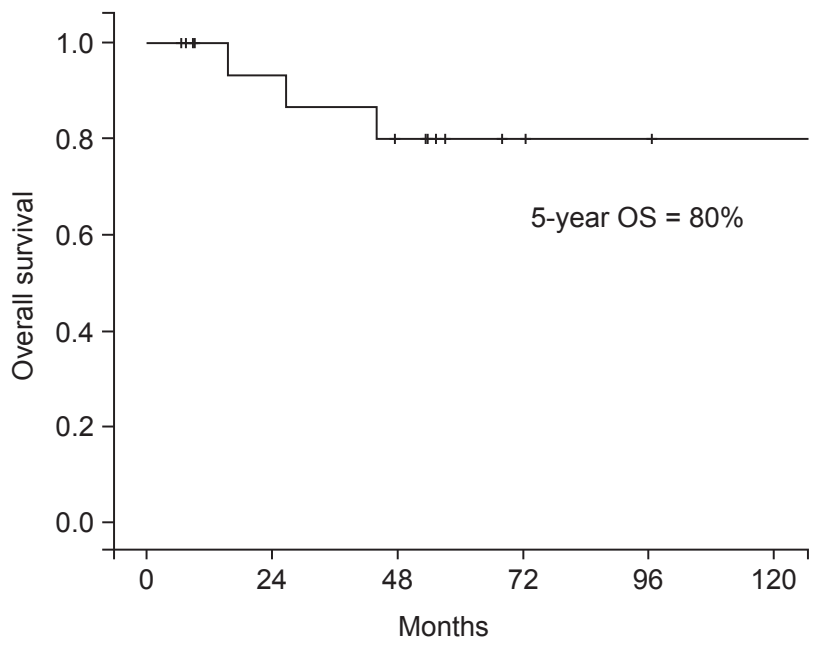

D

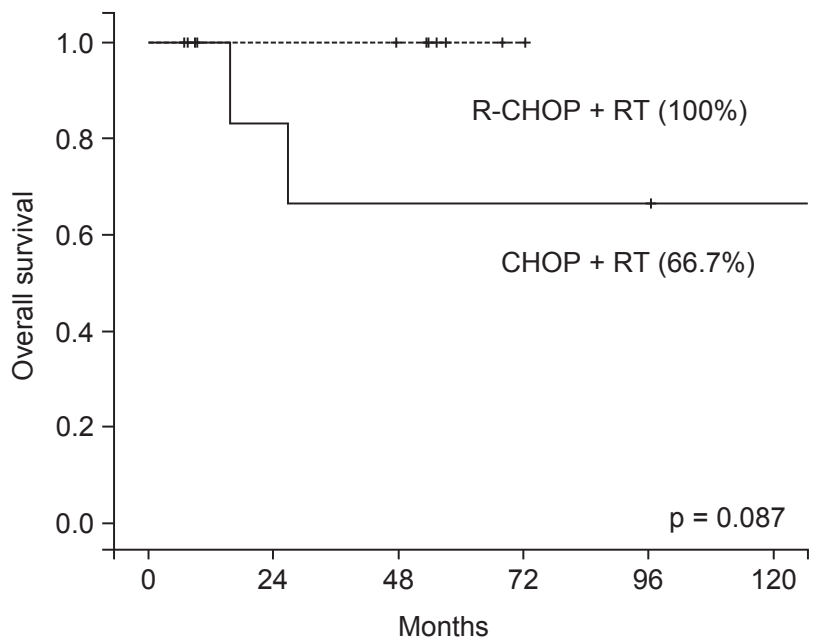

Fig. 2. (A) Progression-free survival (PFS) and (B) overall survival (OS) rates for all patients, and according to the treatment type, $C H O P$ + RT group and R-CHOP + RT group (C, D). CHOP, cyclophosphamide, doxorubicin, vincristine, and prednisone; R-CHOP, rituximab, cyclophosphamide, doxorubicin, vincristine, and prednisone; RT, radiotherapy. 
with NHL [7-9,11]. Laskar et al. [13] demonstrated that diseasefree survival (DFS) and OS rates are higher in patients treated with chemotherapy combined with RT than in those treated with chemotherapy alone. Mohammadianpanah et al. [15] also reported a significantly better DFS rate by using a combined treatment involving chemotherapy and RT, which is consistent with our study.

It has been documented that R-CHOP improves PFS and OS without significant toxic effects [16]. Thus, R-CHOP chemotherapy has been established as the standard treatment for aggressive NHL. Although the role of RT in R-CHOP chemotherapy has been reevaluated, there is no convincing evidence for the necessity of a higher RT dose $[11,12]$. Phan et al. [12] reviewed 44 DLBCL patients treated with $\mathrm{R}-\mathrm{CHOP}$, with or without consolidative RT (30-39.6 Gy), after 6 to 8 cycles of R-CHOP using matched pair analysis. With lower RT doses than those of previous studies, such as SWOG 8736, SWOG 0014, and ECOG 1484, R-CHOP chemotherapy with RT significantly improved the 5-year PFS and OS rates compared to chemotherapy alone. However, to the best of our knowledge, there have been no reports on the role of RT in R-CHOP chemotherapy for tonsillar lymphoma. In our study, we evaluated patients with DLBCL who were treated with R-CHOP (median, 6 cycles) and low-dose consolidative RT (median, $33.3 \mathrm{~Gy}$ ), who demonstrated 5-year PFS and OS rates of $100 \%$ without experiencing significant toxicity. It is suggested that RT may serve a role in R-CHOP chemotherapy era of primary tonsillar lymphoma.

Laskar et al. [13] suggested that a higher RT doses results in significantly better survival in tonsillar lymphoma. The DFS (91\% vs. 59.7\%, $p=0.039$ ) and 0 ( $88.2 \%$ vs. $78.9 \%, p=$ $0.014)$ rates were found to be significantly higher in patients who were irradiated with $\geq 45 \mathrm{~Gy}$. In multivariate analysis, a radiation dose of $<45$ Gy was significantly related to a lower DFS and OS rate [hazard ratio (HR) 9.65, $p=0.005$ and HR 9.89, $p=0.007$ ]. Mohammadianpanah et al. [14] reported that the 5-year cause-specific and relapse-free survival rates were both $100 \%$ in patients with tonsillar lymphoma who were irradiated with 40-50 Gy. However, these studies have certain limitations in terms of interpreting the results because the majority of patients included were treated with CHOP chemotherapy. There have been attempts toward decreasing RT doses because of the RT-related toxicities reported in numerous studies. Lowry et al. [17] reported that there was no significant difference in local control, PFS, or OS between low-dose (30 Gy) and highdose (40-45 Gy) RT in NHL. In our study, patients treated with R-CHOP chemotherapy combined with low-dose (median, 33.3
Gy) RT showed good survival rates. Both of the 5-year PFS and OS rates were $100 \%$. Therefore, RT doses need to be modified in patients receiving chemotherapy regiments with rituximab, as suggested by Phan et al. [12].

Previous studies have indicated that disease stage, IPI score, disease bulkiness, B symptoms, and performance status are prognostic factors for PFS, DFS, or OS $[13,15]$. The majority of our patients had early-stage disease, an IPI score of 0 , no bulky disease, no B symptoms, and a good performance status. Therefore, there was no significant difference in PFS or OS according to these clinical factors. Another limitation of our study was the small number of patients included and the relatively short follow-up period of the R-CHOP chemotherapy group.

In conclusion, the results of this study suggest that chemotherapy (R-CHOP) combined with relatively low-dose consolidative RT may be a safe and effective treatment for primary tonsillar lymphoma. Despite controversy regarding prognosis, patients with primary tonsillar lymphoma generally have favorable outcomes; therefore, high-dose RT is not necessary to improve local control and survival.

\section{Conflict of Interest}

No potential conflict of interest relevant to this article was reported.

\section{References}

1. Lee SJ, Suh CW, Lee SI, et al. Clinical characteristics, pathological distribution, and prognostic factors in nonHodgkin lymphoma of Waldeyer's ring: nationwide Korean study. Korean J Intern Med 2014;29:352-60.

2. Oin $Y$, Shi YK, He XH, et al. Clinical features of 89 patients with primary non-Hodgkin's lymphoma of the tonsil. Ai Zheng 2006;25:481-5.

3. Lopez-Guillermo A, Colomo $L$, Jimenez $M$, et al. Diffuse large B-cell lymphoma: clinical and biological characterization and outcome according to the nodal or extranodal primary origin. J Clin Oncol 2005;23:2797-804.

4. Yahalom J, Illidge T, Specht L, et al. Modern radiation therapy for extranodal lymphomas: field and dose guidelines from the International Lymphoma Radiation Oncology Group. Int $J$ Radiat Oncol Biol Phys 2015;92:11-31.

5. Chang DT, Mendenhall NP, Lynch JW, Morris CG, Olivier KR. Long-term outcomes for stage I-II aggressive non-Hodgkin Iymphoma of Waldeyer's ring. Am J Clin Oncol 2009;32:233-7. 6. Mian M, Ferreri AJ, Rossi $A$, et al. Role of radiotherapy in 
patients with early-stage diffuse large B-cell lymphoma of Waldeyer's ring in remission after anthracycline-containing chemotherapy. Leuk Lymphoma 2013;54:62-8.

7. Miller TP, Dahlberg S, Cassady JR, et al. Chemotherapy alone compared with chemotherapy plus radiotherapy for localized intermediate- and high-grade non-Hodgkin's Iymphoma. N Engl J Med 1998;339:21-6.

8. Horning SJ, Weller E, Kim K, et al. Chemotherapy with or without radiotherapy in limited-stage diffuse aggressive nonHodgkin's Iymphoma: Eastern Cooperative Oncology Group study 1484. J Clin Oncol 2004;22:3032-8.

9. dos Santos LV, Lima JP, Lima CS, Sasse EC, Sasse AD. Is there a role for consolidative radiotherapy in the treatment of aggressive and localized non-Hodgkin lymphoma? A systematic review with meta-analysis. BMC Cancer 2012;12:288.

10. Bonnet C, Fillet G, Mounier N, et al. CHOP alone compared with $\mathrm{CHOP}$ plus radiotherapy for localized aggressive lymphoma in elderly patients: a study by the Groupe d'Etude des Lymphomes de l'Adulte. J Clin Oncol 2007;25:787-92.

11. Persky DO, Unger JM, Spier CM, et al. Phase II study of rituximab plus three cycles of CHOP and involved-field radiotherapy for patients with limited-stage aggressive B-cell
Iymphoma: Southwest Oncology Group study 0014. J Clin Oncol 2008;26:2258-63.

12. Phan J, Mazloom A, Medeiros $\sqcup$, et al. Benefit of consolidative radiation therapy in patients with diffuse large $B$-cel Iymphoma treated with R-CHOP chemotherapy. J Clin Oncol 2010;28:4170-6.

13. Laskar S, Bahl G, Muckaden MA, et al. Primary diffuse large $\mathrm{B}$-cell lymphoma of the tonsil. Is a higher radiotherapy dose required? Cancer 2007;110:816-23.

14. Mohammadianpanah M, Omidvai S, Mosalei A, Ahmadloo N. Treatment results of tonsillar lymphoma: a 10-year experience. Ann Hematol 2005;84:223-6.

15. Mohammadianpanah $M$, Daneshbod $Y$, Ramzi M, et al. Primary tonsillar lymphomas according to the new World Health Organization classification: to report 87 cases and literature review and analysis. Ann Hematol 2010;89:993-1001.

16. Coiffier B, Lepage $E$, Briere J, et al. CHOP chemotherapy plus rituximab compared with $\mathrm{CHOP}$ alone in elderly patients with diffuse large-B-cell lymphoma. N Engl J Med 2002;346:23542.

17. Lowry L, Smith $P$, Qian $W$, et al. Reduced dose radiotherapy for local control in non-Hodgkin lymphoma: a randomised phase III trial. Radiother Oncol 2011;100:86-92. 